\title{
Evaluación de la restauración forestal de una franja deforestada en 1998 en el Bosque Reservado
}

\section{Evaluationoftheforestrestorationof a stripoflanddeforested in 1998 in the Bosque Reservado}

\section{Avaliação da restauraçãoflorestal de umafaixa de terra desmatada em 1998 na Floresta Reservada}

Fernando Honorato Gutiérrez Huamán

Universidad Nacional Agraria de la Selva

Luis Eduardo Ore Cierto

Universidad Nacional Agraria de la Selva

Wendy Caroline Loarte Aliaga ORCID

Consultor Constructor \& Auditor LEOC E.I.R.L

Juan Daniel Oré Cierto ORCID

ONG - Centro de Información y Educación para la Prevención del Abuso de Drogas -

Pucallpa

\section{RESUMEN}

El propósito del trabajo de investigación fue de conocer el proceso de restauración forestal de una franja deforestada en el año 1998 mediante la evaluación de la composición florística y algunos parámetros dasométricos del bosque de un periodo de 16 años; la superficie afectada fue de 2000 m de longitud x 25 m de ancho (5 ha) (franja de línea de alta tensión). Se deforestó totalmente para el paso de la línea de alta tensión. El trabajo se realizó mediante un inventario forestal por muestreo, con parcelas de $25 \mathrm{~m}$ de ancho x $50 \mathrm{~m}$ de longitud, distribuidas al azar (área de muestreo 0.75 has). Se utilizó el método de fajas a lo largo de las torres; dirección norte - sur, se empleó equipos de medición para obtener las observaciones de variables de diámetro a la altura del pecho (Dap) y altura total. Se considero diámetros a partir de $10 \mathrm{~cm}$. Los resultados estimados del área basal promedio por hectárea fueron de $4.7056 \mathrm{~m} 2$, mientras que el volumen total promedio por hectárea fue de $47.99 \mathrm{~m} 3$. En cuanto a su recuperación florística, se encontró 41 especies forestales, 20 familias y 167 individuos por hectárea; la altura total promedio fue de $12.97 \mathrm{~m}$. El índice de valor importancia incluyen 10 especies entre las principales están: Jacaranda copaia, Senefeldera inclinata, Tachigalia cavipes, Inga altísima, 
Aniba perutilis,Inga edulis, Cecropia sciadophylla, Ladembergia micrantha, Ladembergia micrantha, Virola calophylloides,Protium llewelynii,todas ellas indicadores del bosque secundario. Estos resultados demuestran el grado de recuperación del bosque en fajas ocurre rápidamente, a la existencia de fuentes de semillas y la capacidad de regeneración de las especies, dando inicio de una inmediata sucesión secundaria.

Palabras claves: regeneración, natural, bosque, reservado, restauración, pasiva.

\begin{abstract}
The purpose of the research work was to learn about the forest restoration process of a strip deforested in 1998 by evaluating the floristic composition and some dasometric parameters of the forest over a period of 16 years; the affected area was $2000 \mathrm{~m}$ long x $25 \mathrm{~m}$ wide ( $5 \mathrm{ha}$ ) (high tension line strip). It was completely deforested for the passage of the power line. The work was carried out by means of a forest inventory by sampling, with plots of $25 \mathrm{~m}$ wide x $50 \mathrm{~m}$ long, randomly distributed (sampling area $0.75 \mathrm{ha}$ ). The method of strips was used along the towers; north - south direction, measuring equipment was used to obtain the observations of variables of diameter at breast height $(\mathrm{DBH})$ and total height. Diameters of $10 \mathrm{~cm}$ and above were considered. The estimated results of the average basal area per hectare were $4.7056 \mathrm{~m} 2$, while the average total volume per hectare was $47.99 \mathrm{~m} 3$. Regarding its floristic recovery, 41 forest species, 20 families and 167 individuals per hectare were found; the average total height was $12.97 \mathrm{~m}$. The importance value index includes 10 species among the main ones: Jacaranda copaia, Senefeldera inclinata, Tachigalia cavipes, Inga altísima, Aniba perutilis, Inga edulis, Cecropia sciadophylla, Ladembergia micrantha, Ladembergia micrantha, Virola calophylloides, Protium llewelynii, all of them indicators of the secondary forest. These results demonstrate the degree of recovery of the strip forest occurs rapidly, the existence of seed sources and the regeneration capacity of the species, giving rise to an immediate secondary succession.
\end{abstract}

Key words: regeneration, natural, forest, reserved, restoration, passive. 


\section{RESUMO}

O objectivo do trabalho de investigação era conhecer o processo de restauração florestal de uma faixa desmatada em 1998 através da avaliação da composição florística e de alguns parâmetros dasométricos da floresta durante um período de 16 anos; a área afectada era de $2000 \mathrm{~m}$ de comprimento x $25 \mathrm{~m}$ de largura (5 ha) (faixa de linha de alta tensão). Foi totalmente desmatada para a passagem da linha eléctrica. $\mathrm{O}$ trabalho foi realizado através de um inventário florestal por amostragem, com parcelas de $25 \mathrm{~m}$ de largura x $50 \mathrm{~m}$ de comprimento, distribuídas aleatoriamente (área de amostragem 0,75 ha). O método das tiras foi utilizado ao longo das torres; no sentido norte - sul, foi utilizado equipamento de medição para obter as observações das variáveis de diâmetro à altura do peito (DBH) e altura total. Foram considerados os diâmetros a partir de $10 \mathrm{~cm}$. Os resultados estimados da área basal média por hectare foram de 4,7056 m2, enquanto o volume total médio por hectare foi de 47,99 m3. Quanto à sua recuperação florística, foram encontradas 41 espécies florestais, 20 famílias e 167 indivíduos por hectare; a altura média total foi de $12,97 \mathrm{~m}$. O índice de valor de importância inclui 10 espécies entre as principais são: Jacaranda copaia, Senefeldera inclinata, Tachigalia cavipes, Inga altísima, Aniba perutilis, Inga edulis, Cecropia sciadophylla, Ladembergia micrantha, Ladembergia micrantha, Virola calophylloides, Protium llewelynii, todos eles indicadores da floresta secundária. Estes resultados demonstram o grau de recuperação da floresta em faixas ocorre rapidamente, à existência de fontes de sementes e à capacidade de regeneração da espécie, dando início a uma sucessão secundária imediata.

Palavras-chave: regeneração, natural, floresta, reservado, restauração, passivo.

\section{INTRODUCCIÓN}

La situación de la deforestación y degradación del bosque reservado de la Universidad Nacional Agraria de la Selva (UNAS) es una amenaza a su diversidad biológica (Gutiérrez, 2007). Esta misma situación se da también a nivel nacional y mundial; estos bosques tropicales constituyen un componente de mucha importancia para mitigar el cambio climático. Esta experiencia de investigación aborda de forma práctica el manejo de la restauración natural sin intervención 
antrópica. Por lo tanto, mantener su riqueza y estructura original en el tiempo es de importancia. El estudio que se viene realizando contribuirá a conocer los patrones de los bosques fragmentados. También, la restauración natural es una manera de conservar las especies y los bosques (Ledezma, 1999).

El Bosque Reservado de la Universidad Nacional Agraria de la Selva (BRUNAS), se encuentra en un proceso de restauración natural de su ecosistema, y este a su vez influyendo en las mejoras de la diversidad biológica, control de erosión de suelos, generación de microclimas, control del comportamiento de las fuentes de agua en las diferentes épocas del año, entre otras (Marcos, 1996 y Gutiérrez, 2007); pero también es importante indicar que casi todos los año en la época seca (meses de julio a mediados de setiembre), en las partes altas del BRUNAS (partes altas de microcuenca de Asunción Saldaña y quebrada del Águila), se realiza la quema de la vegetación en transición (Pteridiumaquilinum(L.)

Kuhn.,Andropogon bicornisL., etc.), indiscriminadamente por parte de la población aledaña, y no habiendo los recursos y equipos necesarios estos se salen de control, afectando relativamente parte de la vegetación del BRUNAS, por ende afectando la biodiversidad, microclimas, erosión de suelos, etc. Hasta la fecha se ha deforestado aproximadamente el $25 \%$ de la superficie del bosque (50 años que está administrado por la Universidad Nacional Agraria de la Selva) (Gutiérrez, 2007).

Se han realizado muchos intentos de su restauración de las áreas fragmentadas, sin embargo, el aumento de la población aledaña al bosque y el cambio de uso de las tierras forestales para ampliación de infraestructura de la Universidad y continúas quemas, por lo que la diversidad de flora y fauna está disminuyendo con el tiempo, se está afectando también otros servicios ambientales (Gutiérrez, 2007).

Con el fin de implementar estrategias de restauración forestal, es necesario conocer la restauración natural pasiva y activa del Bosque Reservado de la UNAS. Por lo que el objetivo del presente trabajo de investigación es evaluar el nivel de la restauración forestal mediante la composición de especies vegetales, área basal, volumen y especies forestales de mayor representatividad de una franja deforestada. 


\section{METODOLOGÍA}

El presente trabajo de investigación se realizó, en el Bosque Reservado de la Universidad Nacional Agraria de la Selva (BRUNAS). Se encuentra ubicado en el Km 1.5 de la carretera, Tingo María - Huánuco. Geográficamente se encuentra en el departamento de Huánuco, Provincia de Leoncio Prado, distrito RupaRupa.

El clima se caracteriza por ser cálido y húmedo, con temperaturas medias anuales que oscilan alrededor de los $24^{\circ} \mathrm{C}$. La precipitación pluvial supera los 3,000 mm por año, siendo los meses de mayor precipitación de noviembre a marzo. Así mismo la altitud es de 660 m.s.n.m.

De acuerdo a la clasificación de zonas de vida o formaciones vegetales del mundo $\mathrm{y}$ el diagrama bioclimático de Holdridge (1987); Tingo María se encuentra ubicada en la formación vegetal de bosque muy húmedo Pre montano Sub Tropical (bmh PST). Y de acuerdo a las regiones naturales del Perú, según J. Pulgar Vidal, este se encuentra en Selva Alta o Rupa Rupa.

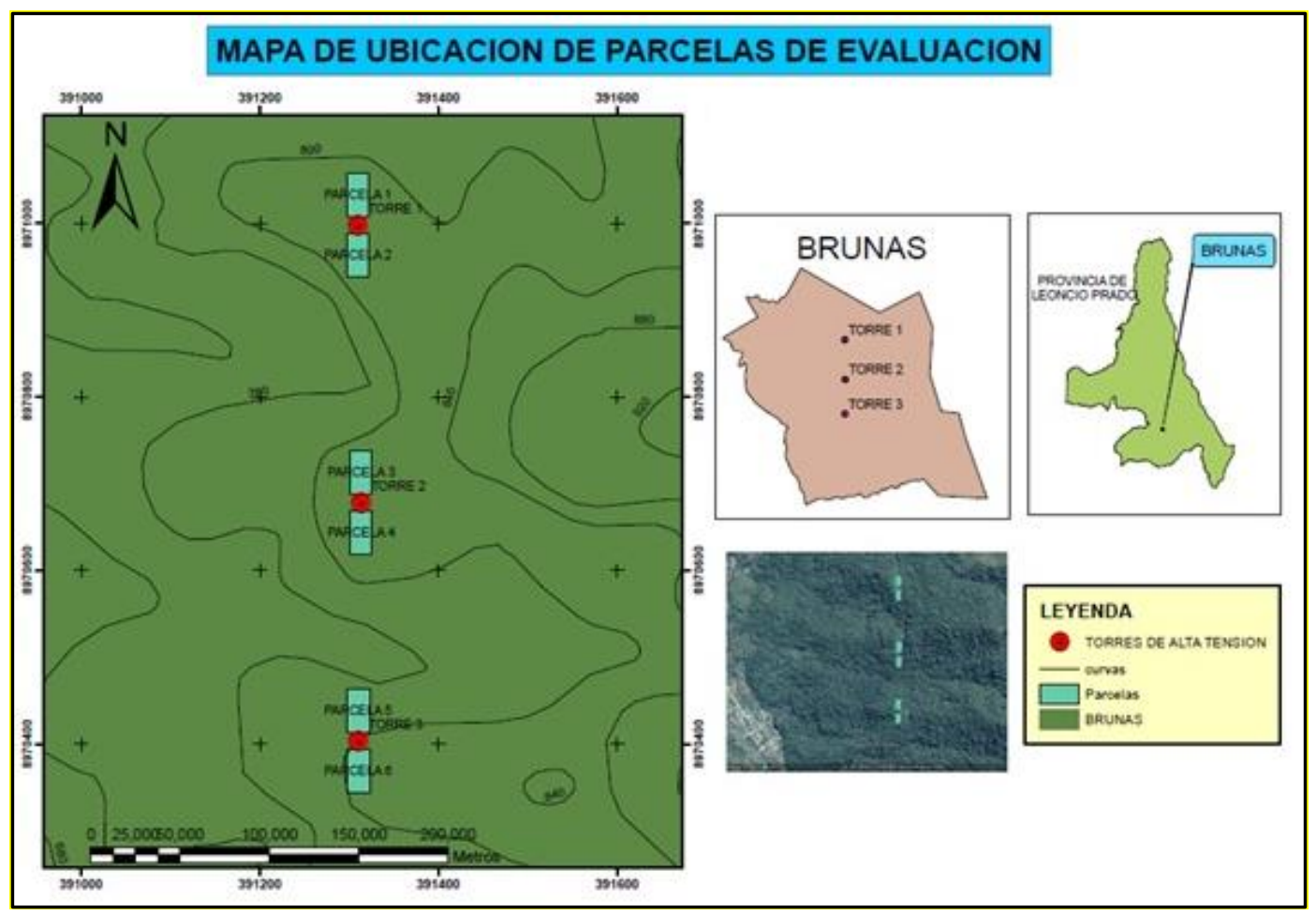

Figura 1. Mapa de ubicación geográfica y distribución de las 6 parcelas de muestreo 


\section{Método de muestreo}

Se realizó mediante un muestreo al azar, en cada parcela se tomó datos de campo, de variables: especies forestales, diámetro, altura total. Se evaluó en parcelas rectangulares de $50 \times 25 \mathrm{~m}$, considerando las especies > de $10 \mathrm{~cm}$ de Dap (Malleux, 1982). Se tomó 6 parcelas de muestreo. Las orientaciones de las parcelas fueron de Norte a Sur. No se consideró las palmeras. Con los datos recopilados se procedió al procesamiento de datos y obtener parámetros del bosque restaurado en forma pasiva ( $\sin$ intervención antrópica) y después de un periodo de 16 años y de esta manera conocer si las especies forestales de los fragmentos pueden mantener su estructura comparada con el bosque original.

Se analizó la composición florística, analizando el número de familias y especies; además, también se determinó el área basal en $\mathrm{m}^{2} / \mathrm{ha}$, volumen en $\mathrm{m}^{3} /$ ha y el Índice de Valor de Importancia en \%, atributos fundamentales para conocer la diversidad forestal; también se determinaron la media, la desviación típica, el error típico y los intervalos de confianza para la media al $95 \%$ de los parámetros en estudio.

\section{RESULTADOS}

En la Tabla 1, se presenta la composición de especies forestales encontradas en la franja revegetada naturalmente. Se encontraron41 especies forestales, 20 familias, siendo las más abundantes las especies de:Jacaranda copaia (Bignonaceae), Senefelderainclinata (Euphorbiaceae), Cecropiasciadophylla (Urticaceae), Inga altísima (Fabaceae), Anibaperutilis (Lauraceae),

Ladembergiamicrantha (Rubiaceae), estimándose un promedio de 162.67 individuos/ ha ( $\geq 10 \mathrm{~cm}$ Dap); en contraste con trabajos de investigación anteriores han encontrado que para el bosque de protección del BRUNAS para una parcela de muestreo de 1.12 ha, tiene: 23 familias, 41 especies, con 303.57 individuos/ ha $(\geq 10 \mathrm{~cm}$ Dap),siendo los más abundantes la Senefelderamacrophylla,

GuatteriadecurrensR.E.Fries, Cecropiasp., Lucumasp. yVirola peruviana (A. DC) Warb (Gutiérrez, 2007); estos resultados demuestran que el futuro dosel de estas especies estánpresentes para el área en proceso de restauración del BRUNAS. 
Tabla1

Composición de especies forestales y número de individuos por ha

\begin{tabular}{|c|c|c|c|c|}
\hline $\mathrm{N}^{\circ}$ & NOMBRE CIENTÍFICO & FAMILIA & NOMBRE COMÚN & $\mathrm{N}^{\circ} \mathrm{ND} / \mathrm{Ha}$ \\
\hline 1 & Scheffleramorototoni & Araliaceae & Aceite caspi & 1.3 \\
\hline 2 & Caryocarglabrum & Caryocaraceae & Almendro colorado & 5.3 \\
\hline 3 & Rollinia peruviana & Annonaceae & Anonilla & 4.0 \\
\hline 4 & Trema micrantha & Ulmaceae & Atadijo & 4.0 \\
\hline 5 & Parkiapendula & Fabaceae & Pashaco colorado & 1.3 \\
\hline 6 & Guarea trichiliodes & Meliaceae & Requia de altura & 1.3 \\
\hline 7 & Cecropia sciadophylla & Urticaceae & Cetico colorado & 9.3 \\
\hline 8 & Miconiabarbeyena & Melastomaceae & Barbeyana & 1.3 \\
\hline 9 & Protium llewelynii & Burceraceae & Copal & 5.3 \\
\hline 10 & Inyantheraparviflora & Myristicaceae & Cumala & 1.3 \\
\hline 11 & Virola calophylloides & Myristicaceae & Cumala amarilla & 5.3 \\
\hline 12 & Inga edulis & Fabaceae & Guaba & 8.0 \\
\hline 13 & Miconiasemulata & Melastomaceae & Rifari colorado & 1.3 \\
\hline 14 & Jacaranda copaia & Bignonaceae & Huamanzamana & 17.3 \\
\hline 15 & Senefeldera inclinata & Euphorbiaceae & Huangana caspi & 16.0 \\
\hline 16 & Eugenia patens & Myrtaceae & Huayabilla & 1.3 \\
\hline 17 & Loreyaracemosa & Melastomaceae & Manzanita & 1.3 \\
\hline 18 & Anibarosaedora & Lauraceae & Moena Rosada & 2.7 \\
\hline 19 & Anibaamazonica & Lauraceae & Moena amarilla & 2.7 \\
\hline 20 & Aniba penutilis & Lauraceae & Moena negra & 8.0 \\
\hline 21 & Manilkarabidentata & Rubiaceae & Nisperillo & 1.3 \\
\hline 22 & Ficus insipida & Moraceae & Oje negro & 2.7 \\
\hline 23 & Triplaris peruviana & Polygonaceae & Tangarana & 1.3 \\
\hline 24 & Jacaratiadigitata & Caricaceae & Papaya caspi & 1.3 \\
\hline 25 & Miconiabiglandulosa & Melastomaceae & Papelillo caspi & 1.3 \\
\hline 26 & Hymenolobiumexcelsum & Fabaceae & Pashaco negro & 1.3 \\
\hline 27 & Albizianiopoides & Fabaceae & Pashaco & 1.3 \\
\hline 28 & Apeibamembranacea & Malvaceae & Peine de mono & 2.7 \\
\hline 29 & Vismiacayennensis & Clusiaceae & Pichirina & 2.7 \\
\hline 30 & Miconiaminutiflora & Melastomaceae & Rifari blanco & 4.0 \\
\hline 31 & Ladembergia micrantha & Rubiaceae & Cascarilla & 8.0 \\
\hline 32 & Inga altisima & Fabaceae & Shimbillo & 9.3 \\
\hline 33 & Hevea brasiliensis & Euphorbiaceae & Shiringa & 2.7 \\
\hline 34 & Cinchonapubescens & Rubiaceae & Sinchona & 5.3 \\
\hline 35 & Sterculiaapetala & Malvaceae & Huarmi caspi & 1.3 \\
\hline 36 & Ochromapyramidali & Malvaceae & Topa & 4.0 \\
\hline 37 & Cedrelingacateniformes & Fabaceae & Tomillo & 1.3 \\
\hline 38 & Guateriamelosma & Annonaceae & Carahuasca & 1.3 \\
\hline 39 & Pourouma bicolor & Urticaceae & Uvilla & 1.3 \\
\hline 40 & Tachigalia cavipes & Fabaceae & Ucshaquiro blanco & 5.3 \\
\hline 41 & Cecropia alba & Urticaceae & Cetico blanco & 4.0 \\
\hline \multicolumn{4}{|c|}{ Total promedio /ha } & 162.67 \\
\hline
\end{tabular}


Revista de investigación científica y tecnológica

Llamkasun

ISSN: 2709-2275 Trabajemos
Artículo científico

Volumen 2, Número 1, enero - junio, 2021

Recibido: 19-01-2021, Aceptado: 15-03-2021
En la Tabla 2, se presenta la estimación del área basal/ha y volumen total, estimado en la franja restaurada después de 16 años, por especies. Obteniéndose el área basal de $4.7056 \mathrm{~m} 2 / \mathrm{ha}$ y Volumen $47.992 \mathrm{~m} 3 / \mathrm{ha}$; en contraste con trabajos de investigación anteriores han encontrado que para el bosque de protección del BRUNAS para una parcela de muestreo de 1.12 ha, tiene un área basal de13.07 $\mathrm{m} 2 /$ hay un volumen total de 150.82 m3/ha (Gutiérrez, 2007); lo que indica que la regeneración o restauración ecológica se encuentra en proceso dinámico, que en el tiempo será capaz de llegar a su estructura original.

Tabla 2

Área basal y volumen total por especies

\begin{tabular}{|c|c|c|c|c|c|}
\hline & NOMBRE & FAMILIA & NOMBRE & $\mathrm{AB} / \mathrm{Ha}$ & VOLT \\
\hline $\mathrm{N}^{\circ}$ & CIENTÍFICO & DE ESPECIE & COMÚN & $(\mathrm{m} 2)$ & $(\mathrm{Ha}) / \mathrm{m} 3$ \\
\hline 1 & Scheffleramorototoni & Araliaceae & Aceite caspi & 0.021 & 0.278 \\
\hline 2 & Caryocarglabrum & Caryocaraceae & Almendro colorado & 0.080 & 0.648 \\
\hline 3 & Rollinia peruviana & Annonaceae & Anonilla & 0.116 & 1.173 \\
\hline 4 & Trema micrantha & Ulmaceae & Atadijo & 0.077 & 0.740 \\
\hline 5 & Parkiapendula & Fabaceae & Pashaco colorado & 0.025 & 0.258 \\
\hline 6 & Guarea trichiliodes & Meliaceae & Requia de altura & 0.013 & 0.085 \\
\hline 7 & Cecropia sciadophylla & Urticaceae & Cetico colorado & 0.191 & 1.343 \\
\hline 8 & Miconiabarbeyena & Melastomaceae & Barbeyana & 0.012 & 0.107 \\
\hline 9 & Protium llewelynii & Burceraceae & Copal & 0.125 & 1.409 \\
\hline 10 & Iryantheraparviflora & Myristicaceae & Cumala & 0.039 & 0.321 \\
\hline 11 & Virola calophylloides & Myristicaceae & Cumala amarilla & 0.160 & 1.817 \\
\hline 12 & Inga edulis & Fabaceae & Guaba & 0.144 & 1.196 \\
\hline 13 & Miconiaserrulata & Melastomaceae & Rifari colorado & 0.017 & 0.142 \\
\hline 14 & Jacaranda copaia & Bignonaceae & Huamanzamana & 0.445 & 4.516 \\
\hline 15 & Senefeldera inclinata & Euphorbiaceae & Huangana caspi & 0.292 & 2.657 \\
\hline
\end{tabular}




\begin{tabular}{|c|c|}
\hline 6 & Eugenia patens \\
\hline 17 & Loreyaracemosa \\
\hline 18 & Anibarosaedora \\
\hline 19 & Anibaamazonica \\
\hline 20 & Aniba perutilis \\
\hline .2 & Manilkarabidentata \\
\hline 2 & Ficus insipida \\
\hline 2 & Triplaris peruviana \\
\hline 2 & Jacaratiadigitata \\
\hline & Miconiabiglandulosa \\
\hline & Hymenolobiumexcelsum \\
\hline$?$ & Albizianiopoides \\
\hline & Apeibamembranacea \\
\hline & Vismiacayennensis \\
\hline & Miconiaminutiflora \\
\hline & Ladembergia micrantha \\
\hline & Inga altisima \\
\hline & Hevea brasiliensis \\
\hline & Cinchonapubescens \\
\hline 2 & Sterculiaapetala \\
\hline & Ochromapyramidali \\
\hline & Cedrelingacateniformes \\
\hline & Guateriamelosma \\
\hline & Pourouma bicolor \\
\hline & Tachigalia cavipes \\
\hline & Cecropia alba \\
\hline
\end{tabular}

Melastomaceae

Lauraceae

Lauraceae

Lauraceae

Rubiaceae

Moraceae

Polygonaceae

Caricaceae

Melastomaceae

Fabaceae

Fabaceae

Malvaceae

Clusiaceae

Melastomaceae

Rubiaceae

Fabaceae

Euphorbiaceae

Rubiaceae

Malvaceae

Malvaceae

Fabaceae

Annonaceae

Urticaceae

Fabaceae

Urticaceae
Huayabilla

Manzanita

Moena Rosada

Moena amarilla

Moena negra

Nisperillo

Oje negro

Tangarana

Papaya caspi

Papelillo caspi

Pashaco negro

Pashaco

Peine de mono

Pichirina

Rifari blanco

Cascarilla

Shimbillo

Shiringa

Sinchona

Huarmi caspi

Topa

Tornillo

Carahuasca

Uvilla

Ucshaquiro blanco

Cetico blanco
0.036

0.020

0.068

0.089

0.280

0.011

0.023

0.061

0.283

0.012

0.040

0.020

0.121

0.069

0.073

0.137

0.360

0.165

0.104

0.044

0.131

0.017

0.025

0.144

0.547

0.063
0.204

0.093

0.634

1.051

2.851

0.071

0.092

0.804

0.054

0.359

0.117

1.331

0.638

0.469

1.243

3.730

2.104

0.868

0.288

0.597

0.081

0.118

2.623

7.389

0.363

Total, promedio /ha

4.703

47.992 
Revista de investigación científica y tecnológica

Llamkasun

ISSN: 2709-2275 Trabajemos

\section{Artículo científico}

Volumen 2, Número 1, enero - junio, 2021

Recibido: 19-01-2021, Aceptado: 15-03-2021

\section{Índice de Valor Importancia (IVI)}

En la Tabla 3, vemos el peso ecológico de las 10 principales especies forestales interactuando de coexistencia y competencia. Nos indica la importancia fitosociológica de una especie dentro de la comunidad arbórea. Siendo las más importantes: Jacaranda
copaia,Senefelderainclinata,

Tachigaliacavipes, Inga altísima, Anibaperutilis, Anibaperutilis, Cecropiasciadophylla,

Ladembergiamicrantha, Virola calophylloides, Protiumllewelynii

Tabla 3

Índice de Valor de Importancia

\begin{tabular}{|c|c|c|c|c|c|c|c|}
\hline $\mathbf{N}^{\circ}$ & $\begin{array}{l}\text { NOMBRE } \\
\text { CIENTÍFICO }\end{array}$ & $\begin{array}{l}\text { FAMILIA } \\
\text { DE ESPECIE }\end{array}$ & $\begin{array}{l}\text { NOMBRE } \\
\text { COMÚN }\end{array}$ & $\begin{array}{l}\text { FREC } \\
\text { REL. }\end{array}$ & $\begin{array}{l}\text { ABU. } \\
\text { REL }\end{array}$ & $\begin{array}{l}\text { DOM. } \\
\text { REL. }\end{array}$ & $\begin{array}{l}\text { IVI } \\
\%\end{array}$ \\
\hline 1 & Jacaranda copaia & Bignonaceae & Huamanzamana & 7.792 & 10.656 & 9.470 & 27.918 \\
\hline 2 & Senefeldera inclinata & Euphorbiaceae & Huangana caspi & 3.896 & 9.836 & 6.209 & 19.941 \\
\hline 3 & Tachigalia cavipes & Fabaceae & Ucshaquiro blanco & 3.896 & 3.279 & 11.625 & 18.799 \\
\hline 4 & Inga altisima & Fabaceae & Shimbillo & 3.896 & 5.738 & 7.655 & 17.289 \\
\hline 5 & Aniba perutilis & Lauraceae & Moena negra & 5.195 & 4.918 & 5.954 & 16.067 \\
\hline 6 & Inga edulis & Fabaceae & Guaba & 5.195 & 4.918 & 3.062 & 13.175 \\
\hline 7 & Cecropia sciadophylla & Urticaceae & Cetico colorado & 2.597 & 5.738 & 4.054 & 12.390 \\
\hline 8 & Ladembergia micrantha & Rubiaceae & Cascarilla & 3.896 & 4.918 & 2.920 & 11.734 \\
\hline 9 & Virola calophylloides & Myristicaceae & Cumala amarilla & 3.896 & 3.279 & 3.402 & 10.577 \\
\hline 10 & Protium llewelynii & Burceraceae & Copal & 3.896 & 3.279 & 2.665 & 9.840 \\
\hline
\end{tabular}

En cuanto a la distribución diamétrica de las especies encontradas nos da la característica de forma de "J" invertida similar a la de un bosque húmedo latifoliado primario $\mathrm{y}$ secundario. La Figura 02 muestra que hay muchos individuos en la clase diamétrica menor, pero a medida que el diámetro aumenta, disminuye el número de individuos, casi en forma logarítmica. Esto nos indica que el bosque es dinámico, corroborándose estadísticamente con el coeficiente de determinación que tiene un valor de 0.9733 , aproximadamente cercano a 1.00. 


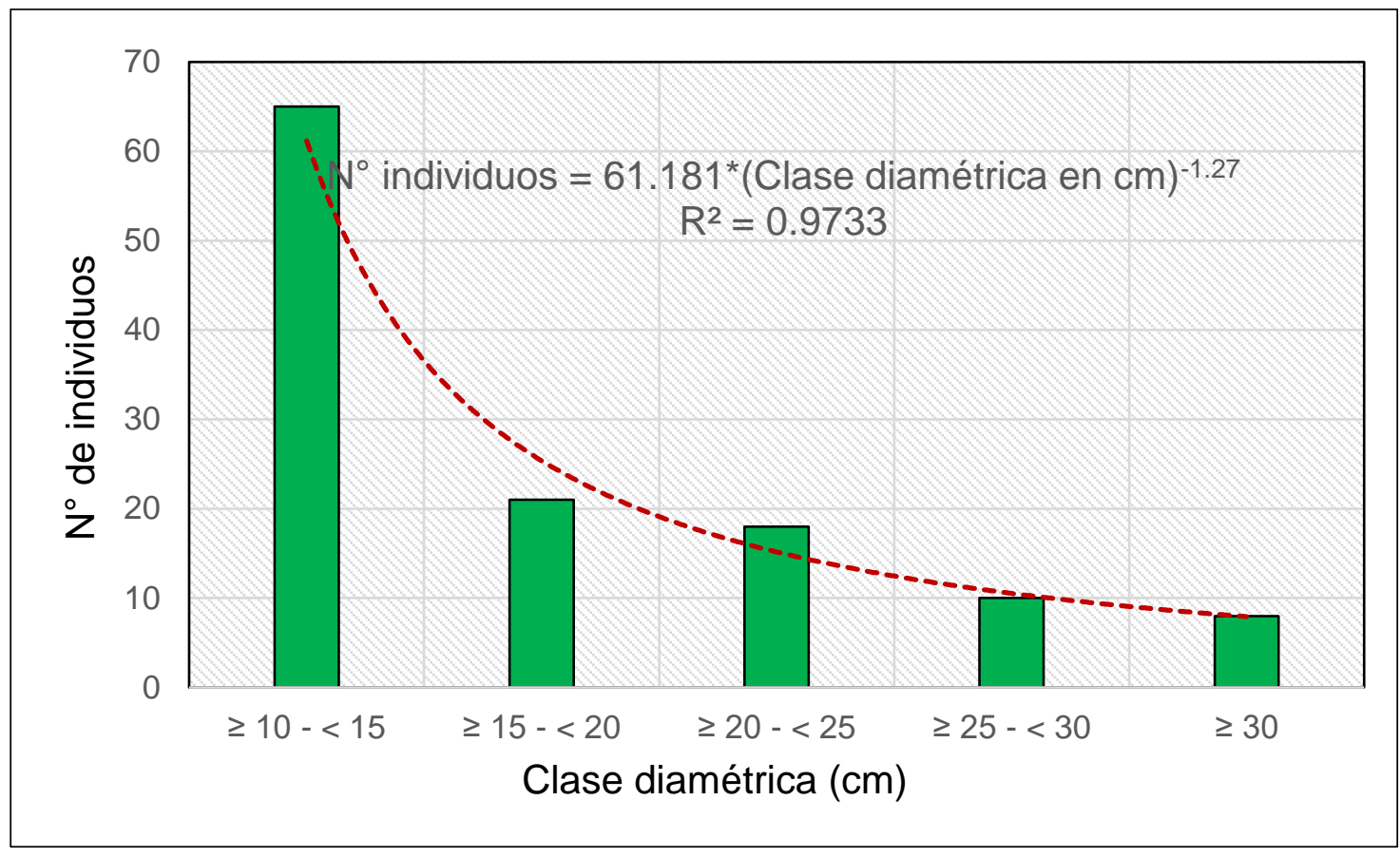

Figura 2. Relación de clase diamétrica y número de individuos

De los resultados podemos interpretar que existe una alta probabilidad de que los parámetros encontrados se acercan a los resultados del bosque de referencia. Individuos $\geq 10 \mathrm{~cm}$ de Dap.

La Tabla 4 muestra los parámetros dasonómicos, donde para el bosque de restauración (BRUNAS) el número de individuos es de 162.67 ind./ha, especies forestales con una riqueza de 41 especies y 20 familias para el área muestreada, con un volumen total de $47.992 \mathrm{~m} 3 / \mathrm{ha}$, y con un área basal de $4.7056 \mathrm{~m} 2 / \mathrm{ha}$; mientras que, en contraste con trabajos de investigación anteriores han encontrado que para el bosque de protección del BRUNAS para una parcela de muestreo de 1.12 ha, tiene: 23 familias, 41 especies, con 303.57 individuos/ ha $(\geq 10 \mathrm{~cm}$ Dap), un área basal de13.07 m2/ha y un volumen total de $150.82 \mathrm{~m} 3 / \mathrm{ha}$ (Gutiérrez, 2007). 
Tabla 4

Comparación del bosque restaurado con un bosque de referencia

\begin{tabular}{|c|c|c|c|c|c|}
\hline Parámetros & $\begin{array}{l}\text { Bosque en } \\
\text { restauración }\end{array}$ & $\begin{array}{l}\text { Bosque de } \\
\text { Producción } \\
\text { BRUNAS* }\end{array}$ & $\begin{array}{l}\text { Bosque de } \\
\text { Protección } \\
\text { BRUNAS* }\end{array}$ & $\begin{array}{l}\text { Bosque } \\
\text { Secundario } \\
\text { Iquitos } * *\end{array}$ & $\begin{array}{l}\text { Bosque Secundario } \\
\text { Chanchamayo } * * *\end{array}$ \\
\hline $\begin{array}{l}\text { Área de parcela de } \\
\text { muestreo en ha }\end{array}$ & 0.75 & 2.84 & 1.12 & 0.95 & 1.00 \\
\hline $\begin{array}{l}\text { Número de Individuos por } \\
\text { ha }\end{array}$ & 162.67 & 309.51 & 303.57 & 579.89 & 694.00 \\
\hline Volumen total en $\mathrm{m}^{3} / \mathrm{ha}$ & 47.992 & 227.23 & 150.82 & 257.08 & 315.80 \\
\hline Especies forestales & 41 & 54 & 41 & 235 & 147 \\
\hline Familias & 20 & 33 & 23 & - & 42 \\
\hline Área basal en $\mathrm{m}^{2} / \mathrm{ha}$ & 4.7056 & 18.62 & 13.07 & 20.18 & 32.39 \\
\hline
\end{tabular}

* Gutiérrez (2007); **Vela (2013); y ***Reynel y Honorio (2012)

Tabla 5

Estadística descriptiva de los parámetros en estudio

\begin{tabular}{|c|c|c|c|c|c|c|}
\hline \multirow{2}{*}{ Tipo de bosque } & \multirow{2}{*}{ Parámetros } & \multirow{2}{*}{ Media } & \multirow{2}{*}{$\begin{array}{l}\text { Desviación } \\
\text { típica }\end{array}$} & \multirow{2}{*}{ Error típico } & \multicolumn{2}{|c|}{$\begin{array}{l}\text { Intervalo de confianza para la } \\
\text { media al } 95 \%\end{array}$} \\
\hline & & & & & $\begin{array}{l}\text { Límite } \\
\text { inferior }\end{array}$ & $\begin{array}{l}\text { Límite } \\
\text { superior }\end{array}$ \\
\hline \multirow{3}{*}{$\begin{array}{l}\text { Bosque en } \\
\text { restauración del } \\
\text { BRUNAS }\end{array}$} & $\begin{array}{l}\text { Número de } \\
\text { Individuos por ha }\end{array}$ & 162.67 & 22.84 & 9.32 & 144.40 & 180.95 \\
\hline & $\begin{array}{l}\text { Volumen total en } \\
\mathrm{m}^{3} / \mathrm{ha}\end{array}$ & 47.992 & 10.633 & 4.341 & 39.484 & 56.501 \\
\hline & Área basal en $\mathrm{m} 2 / \mathrm{ha}$ & 4.7056 & 0.9391 & 0.3834 & 3.9542 & 5.4570 \\
\hline
\end{tabular}

\section{DISCUSIÓN}

Es importante mencionar que las partes altas del BRUNAS se encuentra deforestado con un área aproximadamente de 28.29 ha (Gutiérrez, 2007), suceso ocurrido por la instalación de las antenas de energía eléctrica que pasan por la colina alta del bosque y por las extracciones forestales anteriormente realizadas por los pobladores aledaños (Marcos, 1996); en el área en proceso de restauración del BRUNAS se han encontrado un promedio de 162.67individuos o árboles por hectárea, con un intervalo de confianza 
Revista de investigación científica y tecnológica

Llamkasun

ISSN: 2709-2275 Trabajemos

para la media al 95\% de 144.40 a 180.95 árboles/ha; los 303.57 individuos o árboles/ha reportado (Gutiérrez, 2007) para bosques de protección y los 309.51 individuos o árboles/ha para bosque de producción del plan de ordenación del Bosque Reservado de la UNAS, se ubica fuera de este intervalo; así comolos 694.00 árboles/ha reportados (Reynel y Honorio, 2012) en la diversidad y composición de la flora arbórea en un área de ladera de bosque montano Puya Sacha del valle de Chanchamayo, y los 579.89 árboles/ha reportado (Vela, 2013) en la composición estructural de un bosque primario y un bosque secundario de 12 años en la Reserva Nacional Allpahuayo-Mishana en Iquitos; lo cierto es que, como el área evaluada se encuentra en proceso de restauración ecológica, todavía no ha alcanzado el área deforestada la madurez necesaria, por lo que (Odum, 1983y Freitas, 1996) afirma que la composición florística y la biodiversidad suele ser alta en comunidades de bosque con más edad y sin intervención del hombre, con respecto a los bosques en transición o en sucesión ecológica; además es necesario indicar que las características de los suelos del BRUNAS se encuentran desde extremadamente ácido a fuertemente ácido

\section{Artículo científico}

Volumen 2, Número 1, enero - junio, 2021

Recibido: 19-01-2021, Aceptado: 15-03-2021
(Linares, 2012), por lo que el proceso de restauración tarda más tiempo; y a esto se acompaña, con precipitaciones superiores a los $3300 \mathrm{~mm}$, lavando los suelos desnudos del área deforestada.

El análisis estructural es una de las formas que permite medir el comportamiento del proceso de restauración o del proceso sucesional (Burgos, 1954 y Lombardi, 1979); por lo que de acuerdo a los resultados obtenidos, la vegetación de este bosque sucesional o transicional, se ha encontrado un promedio enárea basal de $4.7056 \mathrm{~m} 2 / \mathrm{ha}$, con un intervalo de confianza para la media al $95 \%$ de $3.9542 \mathrm{~m} 2 /$ ha a $5.4570 \mathrm{~m} 2 / \mathrm{ha}$; los $13.07 \mathrm{~m} 2 /$ ha reportado (Gutiérrez, 2007) para bosques de protección y los $18.62 \mathrm{~m} 2 /$ ha para bosque de producción del plan de ordenación del Bosque Reservado de la UNAS, se ubica fuera de este intervalo; así como los 32.39 m2/ha reportados (Reynel y Honorio, 2012) en la diversidad y composición de la flora arbórea en un área de ladera de bosque montano Puya Sacha del valle de Chanchamayo, y los $20.18 \mathrm{~m} 2 /$ ha reportado (Vela, 2013) en la composición estructural de un bosque primario y un bosque secundario de 12 años en la Reserva Nacional Allpahuayo-Mishana en Iquitos; entonces de acuerdo a los resultados todavía el bosque se 
encuentra en proceso sucesional, conociendo de esta manera la composición y las posibilidades de producción, así mismo como el aseguramiento de su existencia, por lo que también se ha encontrado un promedio en volumen total de $47.992 \mathrm{~m} 3 / \mathrm{ha}$, con un intervalo de confianza para la media al $95 \%$ de $39.484 \mathrm{~m} 3 / \mathrm{ha}$ a $56.501 \mathrm{~m} 3 / \mathrm{ha}$; los 150.82 m3/ha reportado por Gutiérrez (2007) para bosques de protección y los $227.23 \mathrm{~m} 3 / \mathrm{ha}$ para bosque de producción del plan de ordenación del Bosque Reservado de la UNAS, se ubica fuera de este intervalo; así como los $315.80 \mathrm{~m} 3 /$ ha reportados (Reynel y Honorio, 2012) en la diversidad y composición de la flora arbórea en un área de ladera de bosque montano Puya Sacha del valle de Chanchamayo, y los $257.08 \mathrm{~m} 3 / \mathrm{ha}$ reportado (Vela, 2013) en la composición estructural de un bosque primario y un bosque secundario de 12 años en la Reserva Nacional Allpahuayo-Mishana en Iquitos.

Manta (1996), nos indica que las interacciones de coexistencia-competencia de las especies forestales través del Índice de Valor de Importancia (IVI) para el área en proceso de restauración ecológica, determina la importancia de cada especie arbórea en el bosque a través de su presencia (abundancia) y cobertura (área basal y volumen total); por lo que, en la Tabla 03, se aprecia el peso ecológico de las 10 principales especies forestales que están interactuando; entonces estaimportancia fitosociológica de estas especies dentro de la comunidad,de acuerdo a los resultados son: Jacaranda copaia,Senefelderainclinata,

Tachigaliacavipes, Inga altísima, Anibaperutilis, Inga edulis, Cecropiasciadophylla,

Ladembergiamicrantha, Virola calophylloidesyProtiumllewelynii,lo que se puede entender, que estas especies utilizan la mayoría de los recursos energéticos del lugar, y en consecuencia, excluyen a las demás especies que puede estar relacionada con diferentes factores (luz, suelo, etc.).

De la Figura 02, se puede apreciar la distribución diamétrica de las especies encontradas, mostrando un comportamiento de"J" invertida,comportamiento similar a la de un bosque húmedo latifoliado primario;es importante interpretar esta relación, ya que refleja la dinamicidad de un bosque dinámico y estructurado(Lamprecht, 1990); así mismo este autor sostiene que los resultados de los análisis estructurales permiten entre otras cosas, deducciones importantes acerca del origen, las características ecológicas y sinecológicas, el dinamismo y las tendencias 
del futuro desarrollo de las comunidades forestales o vegetales (Ledezma, 1999 y Brienen, 2005); entonces de acuerdo a los resultados obtenidos, se puede observar la buena distribución y estructura de las especies vegetales.

El reclutamiento es un factor importante que puede afectar significativamente la composición y dinámica del bosque. En este caso la franja deforestada no es muy grande (25m). Ambos lados de la franja hay bosques remanentes. Así mismo no hubo intervención humana. De hecho, la abundancia de especies y estructura están en proceso dinámico, como lo demuestran los parámetros obtenidos.

\section{CONCLUSIONES}

El área basal promedio por hectárea en el bosque fragmentado es de $4.7056 \mathrm{~m} 2$ /ha, mientras que el volumen promedio por hectárea es de $47.992 \mathrm{~m} 3 / \mathrm{ha}$, además existen 41 especies, con 20 familias y 162.67 individuos por hectárea; la altura total promedio es 12.97 metro por individuo; y el índice de valor importancia incluyen 10 especies entre las principales están: Jacaranda copaia,Senefeldera inclinata, Tachigalia cavipes, Inga altísima, Aniba perutilis, Aniba perutilis, Cecropia sciadophylla, Ladembergia micrantha, Virola calophylloides, Protium llewelynii

\section{REFERENCIAS BIBLIOGRÁFICAS}

Burgos, J. (1954). Un estudio de la silvicultura de algunas especies forestales en Tingo María, Perú. 56P.

Brienen, R. (2005). Tree Rings in the Tropics: A study on growth and ages of Bolivian rain forest trees. PROMAB, Riberalta, Bolivia. 84P.

Freitas, L. (1996). Caracterización florística y estructural de cuatro Comunidades boscosas de la llanura aluvial inundable en la zona de Jenaro Herrera, Amazonia Peruana. Documento técnico, 21 IIAP, Iquitos Perú. 73P.

Gutiérrez, F. (2007). Plan de Ordenación Forestal del Bosque Reservado de la Universidad Nacional Agraria de la Selva, UNALM, tesis para obtener el grado de magister en Manejo Forestal, $120 \mathrm{P}$.

Lamprecht, H. (1990). Silvicultura en los trópicos. Los ecosistemas forestales en los trópicos y sus especies arbóreas, posibilidades y métodos para un aprovechamiento sostenido. Alemania. $335 \mathrm{pg}$. 
Ledezma, J.C. (1999). Consecuencias de la fragmentación Sobre la Herbivoria de plantas de Ataleaphalerata en la Estación Biológica del Bení. Tesis de grado, Carrera de Biología, Facultad de Ciencias Puras y Naturales. Universidad Mayor de San Andrés, La Paz. 53 P.

Linares, M. (2012). Análisis e interpretación de muestras de suelo del Bosque Reservado de la UNAS. Practica preprofesional - FRNR - UNAS.

Lombardi, L.(1979). Evaluación ecológicasilvicultural de los bosques tropicales. In reunión técnica sobre investigación en plantaciones y manejo de bosques tropicales. INIAA/IICA-OEA. Pucallpa, Perú.

Malleux, J. (1982). Inventarios forestales en bosques tropicales - Universidad Nacional Agraria La Molina. 414P.

Manta, M. (1996). Lineamientos metodológicos para el análisis silvicultural de bosques naturales, con fines de producción maderera. UNALM - PERU.

Marcos, C. (1996). Plan maestro para el establecimiento de un Arboreto en el Bosque Reservado de la Universidad Nacional Agraria de la Selva. Tesis para optar el título de Ingeniero en Recursos Naturales Renovables. Tingo María, Huánuco. 118P.

Odum, E. (1983). Ecología.3raedc. Edit. Nueva editorial Interamericana, S.A. México, D.F.

Puerta, R. (2007). Modelo digital de elevación del Bosque Reservado de la Universidad Nacional Agraria de la Selva, Tingo María - Perú. Tesis de Posgrado de la UNAS. 63 P.

Reynel, C., Honorio, E. (2012). Diversidad y composición de la flora arbórea en un área de ladera de bosque montano Puya Sacha del valle de Chanchamayo. Asociación Peruana para la Promoción del Desarrollo Sostenible APRODES. 391P.

Vela, D. (2013). Composición estructural de un bosque primario y un bosque secundario de 12 años en la Reserva Nacional Allpahuayo-Mishana en Iquitos. Tesis de la Escuela de formación profesional de Ingeniería Forestal. Facultad de Ciencias Forestales - UNAP.

\section{Contacto:}

Fernando Honorato Gutiérrez Huamán Fgutierrez16471@yahoo.com 